\title{
Undercoverage of lateral trochlear resection is correlated with the tibiofemoral alignment parameters in kinematically aligned TKA: a retrospective clinical study
}

Zhiwei Wang ${ }^{1}$, Liang Wen ${ }^{1 *}$ D, Liang Zhang ${ }^{2}$, Desi Ma' ${ }^{1}$ Xiang Dong ${ }^{2}$ and Tiebing $\mathrm{Qu}^{3}$

\begin{abstract}
Background: A mismatch between the femoral component and trochlear resection surface is observed in kinematically aligned total knee arthroplasty (KA-TKA) when conventional prostheses are employed. This mismatch is mainly manifested in the undercoverage of the lateral trochlear resection surface. The aim of the present study was to assess the relationship between the mismatch and the alignment parameters of the tibiofemoral joint.

Methods: Forty-five patients (52 knees) who underwent KA-TKA in our hospital were included. Patient-specific instrumentation was used in 16 patients (16 knees), and conventional instruments with calipers and other special tools were employed in the other 29 patients (36 knees). The widths of the exposed resection bone surface at the middle (MIDexposure) and distal (DISexposure) levels on the lateral trochlea were measured as dependent variables, whereas the hip-knee-ankle angle (HKAA), mechanical lateral distal femoral angle (mLDFA), joint line convergence angle (JLCA), medial proximal tibial angle (MPTA) and transepicondylar axis angle (TEAA) were measured as independent variables. Correlation analysis and subsequent linear regression were conducted among the dependent variables and various alignment parameters of the tibiofemoral joint.

Results: The incidence of undercoverage of the lateral trochlear resection surface was $86.5 \%$ with MIDexposure and DISexposure values of 2.3 (0-6 mm) and $2.0(0-5 \mathrm{~mm})$, respectively. The widths of the two levels of exposed bone resection were significantly correlated with mLDFA and HKAA but were not related to TEAA.

Conclusions: The undercoverage of the trochlear resection surface in KA-TKA is mainly correlated with the degree of valgus of the distal femoral joint line. The current study suggests that this correlation should be considered in the development of KA-specific prostheses.
\end{abstract}

Keywords: Total knee arthroplasty, Osteoarthritis, Kinematic alignment, Trochlea, Anatomy

* Correspondence: wenliang@ccmu.edu.cn

'Department of Orthopedic Surgery, Beijing Chaoyang Hospital, Capital

Medical University, 100020 Beijing, China

Full list of author information is available at the end of the article

C C The Author(s). 2021 Open Access This article is licensed under a Creative Commons Attribution 4.0 International License, which permits use, sharing, adaptation, distribution and reproduction in any medium or format, as long as you give appropriate credit to the original author(s) and the source, provide a link to the Creative Commons licence, and indicate if changes were made. The images or other third party material in this article are included in the article's Creative Commons licence, unless indicated otherwise in a credit line to the material. If material is not included in the article's Creative Commons licence and your intended use is not permitted by statutory regulation or exceeds the permitted use, you will need to obtain permission directly from the copyright holder. To view a copy of this licence, visit http://creativecommons.org/licenses/by/4.0/. The Creative Commons Public Domain Dedication waiver (http://creativecommons.org/publicdomain/zero/1.0/) applies to the data made available in this article, unless otherwise stated in a credit line to the data. 


\section{Background}

Total knee arthroplasty (TKA) can significantly alleviate the pain of patients with end-stage knee osteoarthritis (OA) and improve knee joint function and quality of life $[1,2]$. TKA has achieved tremendous success in the past few decades. In terms of alignment options, mechanical alignment (MA), as a standard surgical technique, has won the approval of the majority of surgeons and technical support from almost all knee prosthesis manufacturers. The aim of MA is to restore the "neutral" alignment of the lower extremities; however, the alignment philosophy of MA conflicts with the "natural" alignment of the normal population [3]. Therefore, some studies supposed that the surgical technical requirements of MA are related to the high dissatisfaction rate of patients after TKA [4-6]. Kinematical alignment (KA) is dedicated to restoring the anatomical geometry of the tibiofemoral joint and the laxity of the joint to that which it would have been before the onset of arthritis [7] and abandoning the "neutral" alignment of the MA. KA has attracted widespread attention in recent years [8-10].

Although kinematically aligned TKA (KA-TKA) strives to restore the morphology of the natural tibiofemoral joint, the use of conventional prostheses to perform KATKA cannot take the patellofemoral joint into full consideration [11]. Therefore, whether KA-TKA can cause unpredictable patellofemoral disorders has been widely studied. A matched case-control study suggests that patellofemoral instability following KA-TKA is related to greater flexion of the femoral component [12]. Some published studies suggest that KA-TKA is more conducive to the restoration of patellofemoral biomechanics than MA-TKA [13, 14], whereas other studies indicate that KA-TKA does not restore the anatomy of the femoral trochlea or increase patellofemoral contact stress $[15,16]$. A study based on computer measurements shows that the correlation between natural patellofemoral anatomical parameters and various tibiofemoral alignment parameters is very poor, suggesting that appreciating patellofemoral anatomy will be an important issue in the design of KA femoral prostheses [17].

The above-mentioned studies on patellofemoral disorders related to KA-TKA do not suggest that MA-TKA can perfectly reconstruct the patellofemoral anatomy [11, 12, 15-17]. In contrast, given MA-TKA's inherent limitations, the distal lateral femoral condyle might be overstuffed, which increases the tension of the lateral retinaculum and is associated with the risk of patellar maltracking [18]. A virtual MA-TKA study suggests that the anterior flange of the femoral component should be lateralized to optimize femoral bone coverage and to eliminate the anteromedial overhang [19]. Another study using virtual resection showed that the geometries of the
"Grand piano sign" of anterior femoral resection were different between KA-TKA and MA-TKA, and the ratios of the heights of medial and lateral trochlear resection were approximately 0.7 and 0.6 , respectively [20].

To our knowledge, no study has focused on the mismatch between the femoral component and the trochlear resection surface when KA-TKA is performed using conventional prostheses. In clinical practice, we found that undercoverage of the lateral trochlear resection surface in KA-TKA is more common than that in MATKA. We hypothesized that this scenario was caused by the design of conventional prostheses, which were developed to comply with the requirements of MA. The reference axes of KA-TKA however are completely different from those of MA-TKA. We hypothesized that the alignment parameters of the lower limbs of different individuals, especially the joint line orientation and the rotation alignment parameters of the distal femur, would have an impact on this undercoverage. Therefore, the aim of the present study was to investigate the correlation between various tibiofemoral alignment parameters and the undercoverage of lateral trochlear resection.

\section{Methods \\ Patients}

The Kellgren-Lawrence classification system was used to classify knee OA for each case [21]. Patients with KL grade III or grade IV in the tibiofemoral joint as well as patients with $\leq 5$ degrees varus of the proximal tibia (medial proximal tibial angle $\geq 85$ degrees), $\leq 5$ degrees of valgus deformity were considered for KA-TKA. Exclusion criteria included inflammatory arthritis, previous intra-articular fracture, previous collateral ligament or posterior cruciate ligament rupture, genu recurvatum, and fixed flexion contracture greater than 20 degrees. Given that trochlear dysplasia may have unknown impacts on this study, patients with trochlear dysplasia found preoperatively or intraoperatively were also excluded from this retrospective study.

From May 2018 to August 2020, a total of 55 patients underwent KA-TKA in our institution. After excluding three patients diagnosed with trochlear dysplasia and seven patients with missing intraoperative measurement data of lateral trochlear resection exposure, 45 patients (52 knees) were finally included in this study. Eleven patients were men, and 34 patients were women. The mean age was $69.5 \pm 6.7$ years (56-85 years), and the mean body mass index was $28.6 \pm 4.2 \mathrm{~kg} / \mathrm{m}^{2}\left(26-34 \mathrm{~kg} / \mathrm{m}^{2}\right)$. The preoperative radiographic changes of 1 knee were in accordance with Kellgren-Lawrence grade III, and the other 51 knees were in compliance with grade IV. Fifty patients had varus knees, and 2 patients had valgus knees. Among them, 16 patients (16 knees) underwent KA-TKA with the assistance of patient-specific instrumentation (PSI-KA), 
and 29 patients (36 knees) underwent KA-TKA using conventional instruments with measurement tools (Calipered kinematically aligned instrumentation, Calipered-KA). All bilateral KA-TKAs (seven patients) were performed using the Calipered-KA technique.

\section{Surgical plans}

The design of PSI was based on full-length computed tomography (CT) of the lower extremities, whereas the surgical technique of calipered KA followed the technique recommended by Howell et al. [22]. Regardless of which assistant alignment instrument was used, an articular surface-based bone cut approach was adopted [9]. A Vernier caliper was used to measure the thickness of the resected bone pieces of the distal femoral condyles, posterior condyles, and tibial plateau. The general principle is that the sum of the thickness of the resected bone piece, the compensated thickness of the worn cartilage, and the width of the saw kerf is equal to the thickness of the component.

To manufacture PSI, CT data (slice thickness, 0.625 $\mathrm{mm}$ ) were collected and imported into Mimics (version 17, Materialise NV, Belgium) for 3D reconstruction. Then, the solid models were imported into NX 9.0 (Siemens PLM Software, TX, US) for the design of PSI (Fig. 1). Rapid prototyping technology (Formiga P 110, EOS, Krailling, Germany) was used for 3D printing of the PSI. The printing material was medical nylon (PA2200 Polymer powder, EOS, Krailling, Germany), which can be sterilized using autoclaving.

For the intraoperative application of PSI, all the residual articular cartilage should be removed using a curette before the PSI is secured to its unique position. In contrast to the management of articular cartilage in PSI-KA, calipered KA only removed the residual cartilage on the severely worn side. If the contralateral articular cartilage was intact, then it was kept in place. Stacked neodymium magnets ( $1 \mathrm{~mm}$ of thickness each) were used to compensate for the cartilage thickness on the severely worn side (Fig. 2). An intramedullary rod was introduced to ensure medial-lateral and flexionextension orientation of the distal femoral cutting jig. An extramedullary referencing jig was applied on the tibia side.

The distal femoral resection was parallel to the joint line of the distal femur, and the posterior condylar resection was parallel to the posterior condylar axis (PCA). The 'posterior referencing' technique was used in femoral bone preparation. The tibial plateau resection was based on the original inclination of the proximal tibial joint line in the coronal plane. The tibial rotation alignment was consistent with the anteroposterior axis of the lateral plateau, and the posterior tilt of resection was consistent with the posterior slope of the medial plateau. A single posterior cruciate retained (CR)-designed prosthesis (Gemini MK II, Link, Hamburger, Germany) was used in the current study. When the undercoverage of the lateral trochlear resection is too obvious, depending on the coverage of distal femoral resection, the femoral component is appropriately lateralized to alleviate the undercoverage of the lateral trochlea. The patella was not resurfaced in any case.

\section{Parameters measurement}

Before KA-TKA, full-length weight-bearing radiographs of the lower limbs were obtained from all patients. The hip-knee-ankle angle (HKAA) was measured from the full-length radiograph. HKAA is defined as the angle between the mechanical axes of the femur and the tibia. The value of varus HKAA is defined as positive, and the value of valgus HKAA is defined as negative. Other alignment parameter measurements followed the

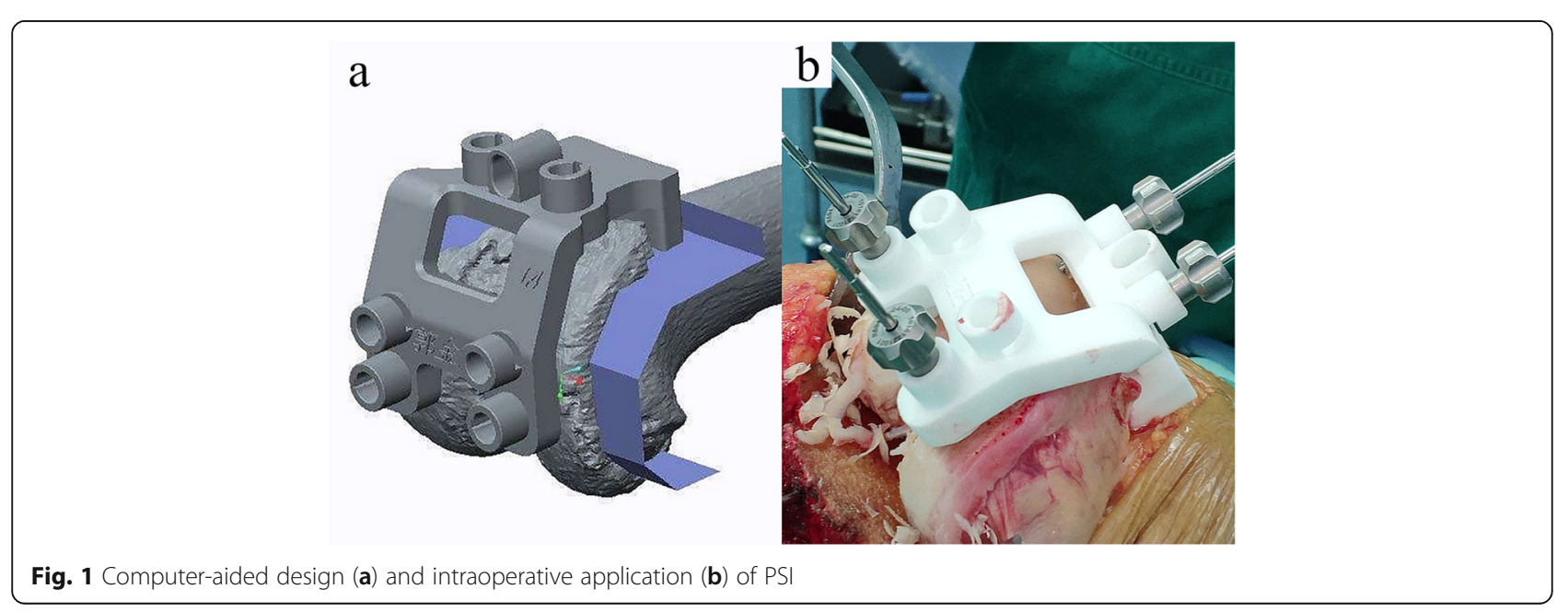




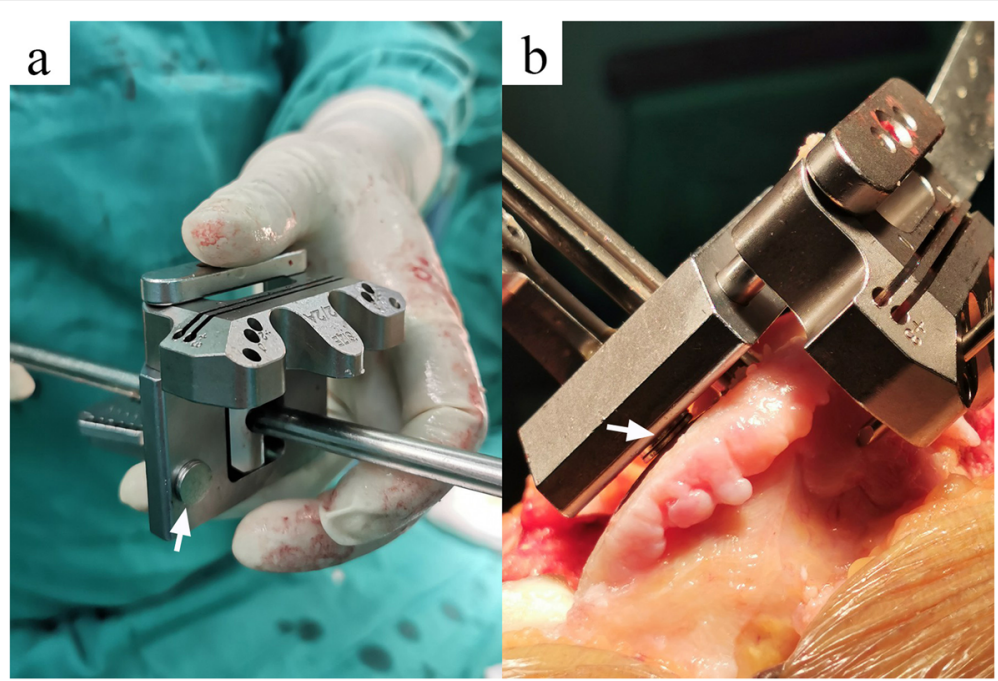

Fig. 2 Stacked neodymium magnets (white arrows) are used to compensate for the thickness of worn articular cartilage (2 mm) in calipered KA

methods described by Paley [23]. The mechanical lateral distal femoral angle (mLDFA), medial proximal tibial angle (MPTA), and joint line convergence angle (JLCA) were measured and recorded (Fig. 3). The full-length CT data of all PSI-KA patients were retrieved. The angle between the surgical transepicondylar axis (TEA) and the posterior condylar axis (PCA) was determined using inhouse maximum intensity projection (MIP) technology (SOMATOM Sensation, Siemens, Germany), and this angle was defined as the transepicondylar axis angle (TEAA) (Fig. 4a). In addition, TEAA was also measured and recorded using a protractor in all patients included in this study during the operation (Fig. 4b). The above parameters were used as independent variables in this study.

The distances from the lateral edge of the trochlear resection surface to the femoral component trial were measured at two levels: the corner of the anterior resection and the anterior chamfer resection (distal exposure, DISexposure) and the middle level between this corner and the apex of the anterior flange of the femoral trial (middle exposure, MIDexposure) (Fig. 5). The widths of the exposed trochlear resection at

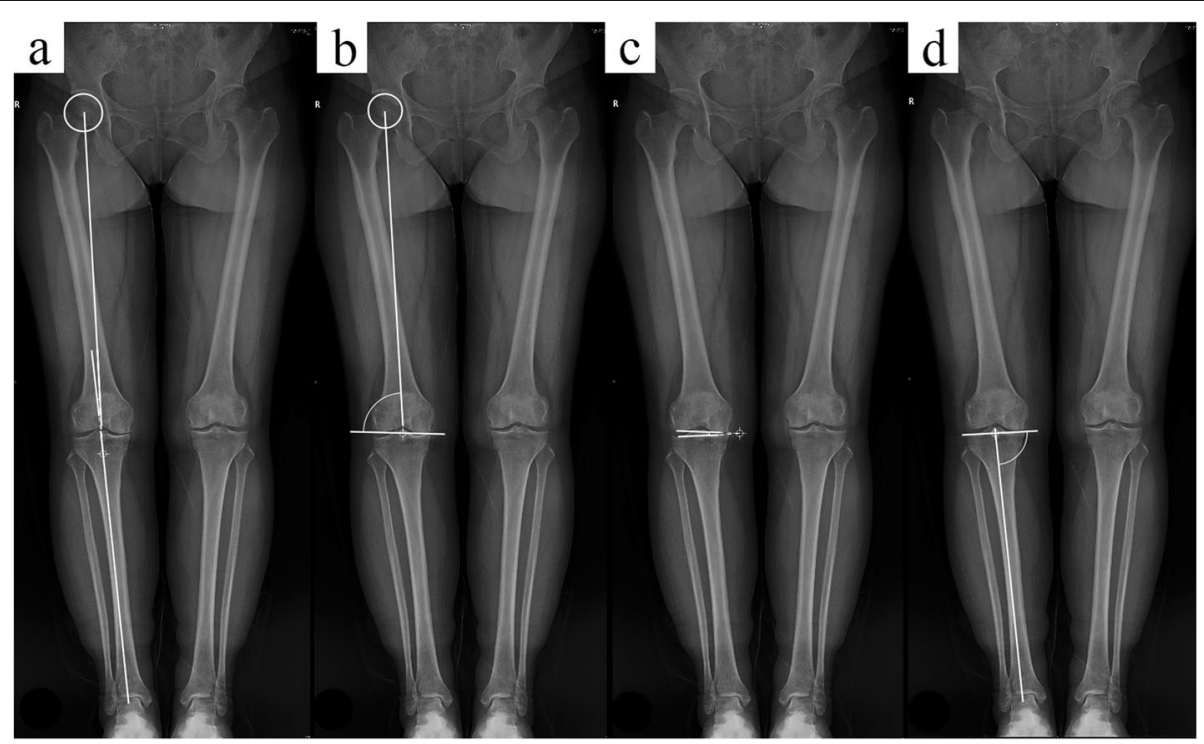

Fig. 3 Schematic diagrams for the measurement of tibiofemoral joint anatomical parameters: a Hip-knee-ankle angle (HKAA), b Mechanical lateral distal femoral angle (mLDFA), $\mathbf{c}$ Joint line convergence angle (JCLA), and $\mathbf{d}$ medial proximal tibial angle (MPTA) 


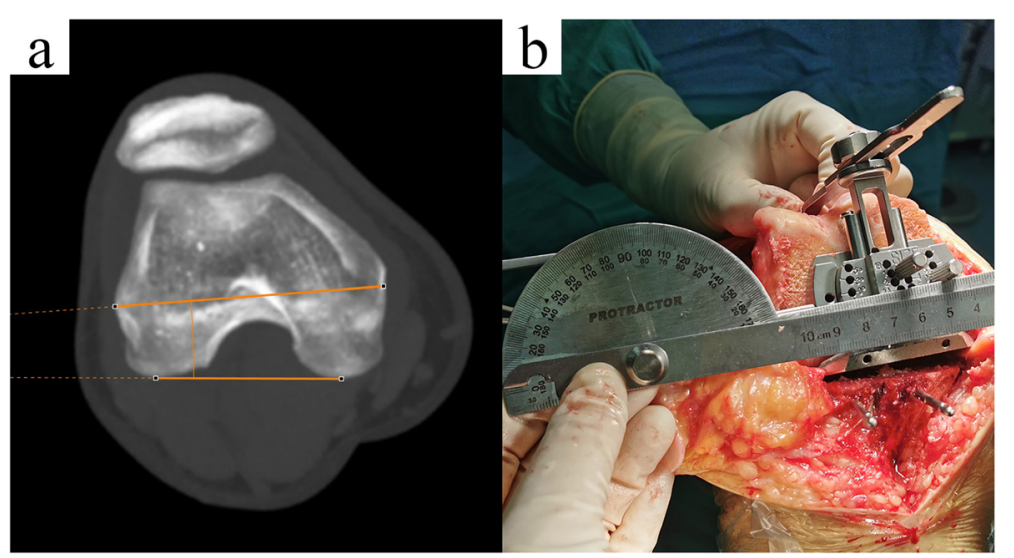

Fig. 4 Transepicondylar axis angles (TEAAs) were measured by preoperative superimposed $C T$ (a) and a protractor in the operating room (b). a Determination of the recess of the medial epicondyle, the prominence of the lateral epicondyle, and the highest point of the medial and lateral posterior condyles by the MIP approach of CT. The TEAA value was automatically obtained by the in-house program. b Intraoperative measurement required electrocautery to mark the position of the medial and lateral epicondyles on the distal femoral resection surface. The arms of the protractor were parallel to the posterior pedals of instruments and the electrocautery marks

these two levels were used as the dependent variables for subsequent analysis.

\section{Statistical analysis}

All measurement parameters were subjected to the Shapiro-Wilk test. Parameters that conformed to a normal distribution are presented as the mean \pm standard deviation, and those that did not conform to a normal distribution are presented as the median (interquartile

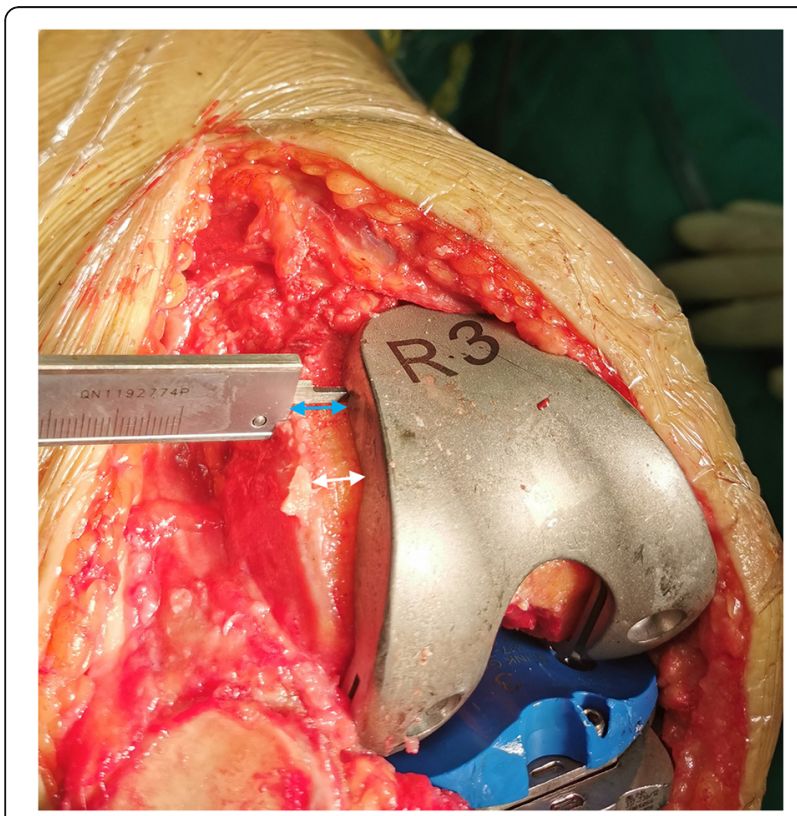

Fig. 5 Intraoperative caliper measurement of the exposed bone resection of the lateral trochlea. The white and blue arrows represent the width of the exposed resection at the distal (DISexposure) and middle (MIDexposure) levels of the trochlea, respectively range). In patients receiving PSI-KA, a reliability test of the two sets of TEAA data from CT measurements and intraoperative measurements was conducted. Reliability was determined by calculating the intraclass correlation coefficient (ICC) with a $95 \%$ confidence interval (CI). An ICC value of $>0.8$ indicates very good, $0.6-0.8$ good, $0.4-0.6$ moderate, and $<0.40$ poor[24]. A correlation test was performed between the dependent variables and all independent variables, and linear regression analyses were conducted between variables with significant correlations. All analyses were performed using SPSS (v. 22.0, IBM, Armonk, NY), and $P<0.05$ was considered statistically significant.

\section{Results}

The mLDFA, MPTA and TEAA conformed to a normal distribution, whereas the other parameters did not. The exposed widths of the distal and middle levels of the lateral trochlear resection surface were $2.0(2.4) \mathrm{mm}(0 \sim 5$ $\mathrm{mm})$ and $3.0(2.9) \mathrm{mm}(0 \sim 6 \mathrm{~mm})$, respectively. The other measurements of the respective variables are shown in Table 1 . Only 7 knees out of 52 knees had no obvious bone resection exposure of the lateral trochlea, and other knees had various extents of lateral trochlear resection exposure (incidence rate $86.5 \%$ ).

The reliability test (ICC, two-way mixed effects, random, fixed effects) of the two sets of TEAA measurements was performed in 16 patients who received PSI$\mathrm{KA}$, and the ICC was 0.792 (0.501-0.922), indicating that the two measurement approaches had good consistency. For the data collection of TEAA, the MIP approach of CT exhibits better accuracy than 
Table 1 Descriptive statistics of various measurement parameters

\begin{tabular}{llll}
\hline & Shapiro-Wilk (Sig.) & Mean \pm SD (Range) & Median (Interquartile range) (Range) \\
\hline HKAA $\left(^{\circ}\right)$ & 0.001 & NA & $6.0(3.0)(-3.2 \sim 9.6)$ \\
mLDFA $\left(^{\circ}\right)$ & 0.658 & $88.2 \pm 2.0(83.7 \sim 93)$ & NA \\
JLCA $\left(^{\circ}\right)$ & 0.000 & NA & $4.9(1.8)(-4.9 \sim 7.4)$ \\
MPTA $\left(^{\circ}\right)$ & 0.466 & $87.2 \pm 1.3(85 \sim 90.7)$ & NA \\
TEAA $\left(^{\circ}\right)$ & 0.093 & $3.4 \pm 1.2(1 \sim 6)$ & NA \\
DISexposure $(\mathrm{mm})$ & 0.035 & NA & $2.0(2.4)(0 \sim 5)$ \\
MIDexposure $(\mathrm{mm})$ & 0.023 & NA & $3.0(2.9)(0 \sim 6)$ \\
\hline
\end{tabular}

NA Not applicable

intraoperative measurement [25], so TEAA data of PSIKA patients are all derived from CT measurements.

Spearman correlation analysis results showed that the two levels of lateral trochlear resection exposure were significantly correlated with mLDFA and HKAA but not correlated with other independent variables (Table 2). Further linear regression analyses showed that DISexposure and MIDexposure are more sensitive to the values of mLDFA with regression coefficients of $-0.480\left(R^{2}=\right.$ $0.496)$ and $-0.724\left(R^{2}=0.702\right)$, respectively, whereas HKAA has a slight influence on the exposure of the trochlear resection surface. The regression coefficients were $-0.310\left(R^{2}=0.357\right)$ and $-0.384\left(R^{2}=0.340\right)$, respectively (Fig. 6).

\section{Discussion}

The current study confirmed that the extent of exposed lateral trochlear resection during KA-TKA has a correlation with mLDFA. Thus, that the more valgus the joint line of the distal femur is, the larger the lateral trochlear resection surface is exposed. In addition, the extent of exposed lateral trochlear resection is also weakly correlated with the alignment of the lower extremities: the more severe the varus knee is, the smaller the exposed resection surface of the trochlea. The results of this study suggested that the design of a new prosthesis that meets the technical requirements of KA might require lateralization of the anterior flange of the femoral component to a certain extent. Due to significant variation in mLDFA among individuals, such lateralization adjustment should be meticulous; otherwise, in some patients, it could result in lateral overhang of the anterior flange,

Table 2 Nonparametric Spearman's correlation results among measurement parameters

\begin{tabular}{llllll}
\hline & & HKAA & mLDFA & JLCA & MPTA \\
\hline MIDexposure & Correlation Coefficient & -0.636 & -0.834 & 0.049 & 0.129 \\
& $P$ (2-tailed) & $<0.01$ & $<0.01$ & 0.732 & 0.363 \\
DISexposure & Correlation Coefficient & -0.646 & -0.689 & -0.064 & 0.242 \\
& P (2-tailed) & $<0.01$ & $<0.01$ & 0.652 & 0.084 \\
\hline
\end{tabular}

irritation of the lateral retinaculum and patellar maltracking. The results of this study implied that personalized custom prostheses or modular femoral components may be more promising for the restoration of patellofemoral anatomy in KA-TKA.

Many factors may be related to the undercoverage of the lateral trochlear resection during KA-TKA, such as the joint line orientation of the distal femur (i.e., mLDFA), the rotational alignment of the femoral component (i.e., TEAA), the flexion of the femoral component, the size of the femoral component, the ratio aspect of the distal femur, and other limb alignment parameters (i.e., HKAA, JCLA, MPTA). Among them, the role of mLDFA is the most reasonable. Brar et al. reported that the apex of the anterior flange moves $4 \mathrm{~mm}$ medially on average in KA-TKA compared with MA-TKA [26]. Given the valgus joint line of the distal femur in most patients, the femoral component requires more internal rotation on the coronal plane compared with MA-TKA, and then the anterior flange tilts medially and exposes the lateral trochlear resection.

Before this study, we hypothesized that TEAA would also affect the undercoverage of lateral trochlear resection. In terms of femoral component rotation alignment, the reference axes in KA-TKA and MA-TKA are the primary femoral axes (i.e., cylindrical axis) and TEA, respectively. The primary femoral axis is the connecting line between the centers of best-fit spheres of medial and lateral condyles [27], whereas TEA is the most reliable reference axis in the measured resection technique of MA-KTA. The primary femoral axis has been proven to be relatively internally rotated compared with TEA [28]. The rotational alignment of the femoral component significantly affects patellofemoral kinematics; an average $5^{\circ}$ of rotation of the femoral component results in a $4^{\circ}$ tilt of the patella [29]. However, the results of this study were beyond the preoperative speculation: the extent of exposed trochlear resection is not significantly related to TEAA. This finding indicates that the rotational alignment of the femoral component does not significantly affect the match between the anterior flange and trochlear resection surface. This result may be explained by 

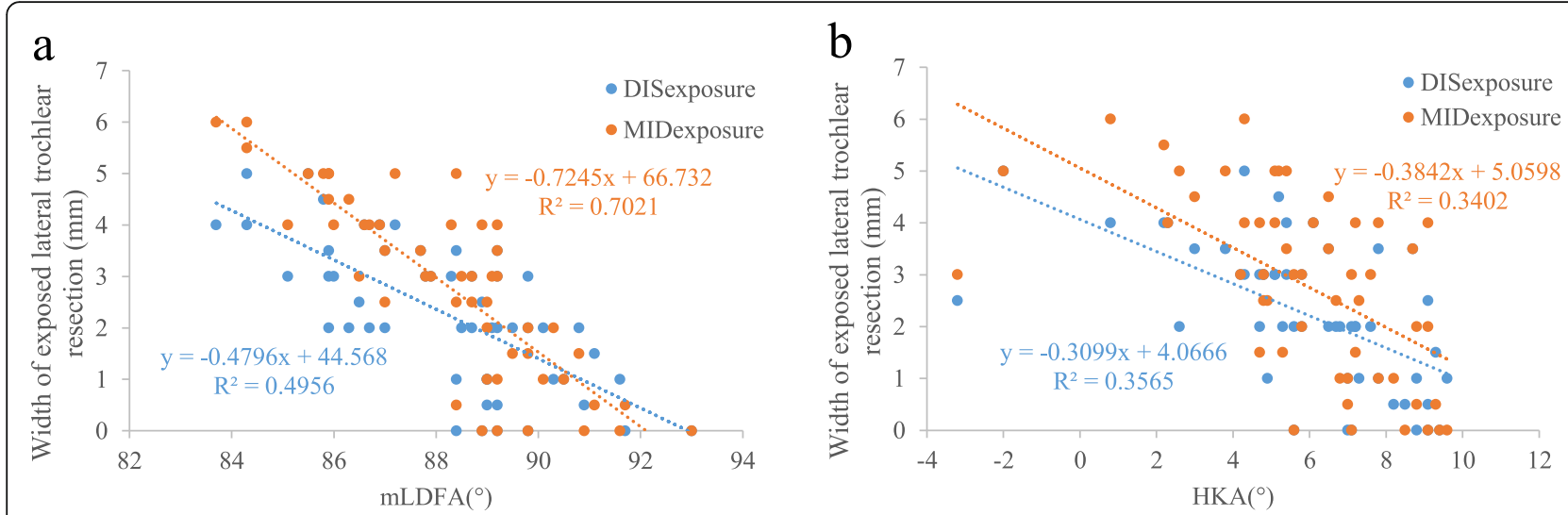

Fig. 6 The influence of the distal femoral joint line (mLDFA) (a) and the alignment of the lower extremities (HKAA) (b) on the undercoverage of lateral trochlear resection, where DISexposure and MIDexposure represent the width of the exposed bone resection at the distal and middle levels of the trochlea, respectively

the fact that the relative internal rotation of the femoral component will only lead to a relative decrease in the amount of lateral trochlear resection rather than significant medialization of the anterior flange.

This study also suggests that HKAA has a minor effect on the undercoverage of the lateral trochlea. HKAA is not only related to the joint line orientation of the distal femur and proximal tibia but is also determined by cartilage loss and soft tissue laxity, which may partially explain why the extent of exposed resection is weakly correlated with the alignment of the lower extremities.

The flexion and the size of the femoral component will also affect the exposure of trochlear resection. Computer simulation analysis shows that the flexion placement of femoral components will cause patellofemoral understuffing, which will increase the width of the trochlear resection $[12,26]$. In the calipered KA group of our study, an intramedullary rod was introduced as deep as possible to ensure the flextion-extension orientation so that the position of the femoral component was compliant with the morphology of the distal femur to avoid excessive flexion of the femoral component. Additionally, downsizing of the femoral component results in anterior cortex notching, which subsequently widens trochlear resection. Although Marra et al. demonstrated that downsizing combined with flexing of the femoral component can eliminate anterior cortex notching without bringing additional risks [30], we still double-checked using the 'angel wing' before bone cutting.

The mismatch of the femoral component aspect ratio has been widely considered in TKA [31]; this issue is of course no exception in KA-TKA. In this study, if undercoverage was found both in distal femoral resection and lateral trochlear resection, then the femoral component was appropriately lateralized to reduce the extent of lateral trochlear resection undercoverage. From the patellofemoral reconstruction perspective, although lateralization of the femoral component could alleviate the undercoverage of the exposed resection, the adjustment extent of such lateralization is very limited because the coverage of distal femoral resection must be taken into account simultaneously. Concerning irritation of the lateral retinaculum, an oscillating saw was used to trim the prominent trochlear ridges in some patients.

There were several limitations in this study. First, only one type of prosthesis was employed in this study, which does not mean that other commercial prostheses have the same extent of trochlear resection exposure. Further clinical studies involving multiple prostheses or computer simulation studies are necessary to confirm the current results. Second, not all patients had CT data in this study. The majority of TEAA data were acquired from intraoperative measurements, which might impact the accuracy of TEAA. However, the preoperative CT data were retrospectively collected from 16 patients who received PSITKA, and the reliability test result of the two sets of TEAAs was "good". Third, the impact of trochlear resection undercoverage on intraoperative patellofemoral biomechanics, such as patellar tracking (no thumb test, positive or negative) or lateral retinaculum release (yes or no), will provide more valuable information for clinical practice. However, the intraoperative assessment of patellofemoral biomechanics only has binary variables, and the number of patients in the present study is not enough to provide sufficient test power for statistical analysis, including categorical variables. Despite the abovementioned limitations, the results of this study are sufficient to demonstrate that the undercoverage of the trochlear resection surface in KA-TKA is not only related to the design philosophy of the conventional prostheses but also correlated with the degrees of valgus of the distal femur. 


\section{Conclusions}

The undercoverage of the trochlear resection surface in KA-TKA is correlated with the degrees of valgus of the distal femoral joint line. The present study suggests that attention should be paid to the impact of tibiofemoral alignment parameters on the match between the femoral component and femoral trochlea resection in the development of KA-specific prostheses.

\begin{abstract}
Abbreviations
KA-TKA: Kinematically aligned total knee arthroplasty; MIDexposure: The exposed resection surface at the middle level on the lateral trochlea; DISexposure: The exposed resection surface at the distal level on the lateral trochlea; HKAA: Hip-knee-ankle angle; mLDFA: Mechanical lateral distal femoral angle; JLCA: Joint line convergence angle; MPTA: Medial proximal tibial angle; TEAA: Transepicondylar axis angle; TKA: Total knee arthroplasty; OA: Osteoarthritis; MA: Mechanical alignment; KA: Kinematical alignment; MA-TKA: Mechanically aligned total knee arthroplasty; PSI: Patient-specific instrumentation; Calipered-KA: KA-TKA using conventional instruments and caliper; CT: Computed tomography; PCA: Posterior condyle axis; CR: Cruciate retained; TEA: Transepicondylar axis; MIP: Maximum intensity projection; ICC: Intraclass correlation coefficient; Cl: 95\% confidence interval
\end{abstract}

\section{Acknowledgements}

Not applicable.

\section{Authors' contributions}

ZW and LW participated in the design of the study, ZW, LW, DM and TQ performed the surgery and took the measurements, LZ and XD participated in the design of PSI and CT measurement, ZW and LW conducted the statistical analyses, ZW and TQ drafted the manuscript. All authors read and approved the final manuscript.

\section{Funding}

The National Natural Science Foundation of China (Grant No. 81572180) provided financial support for the cost of CT and the design of PSI in this study.

\section{Availability of data and materials}

The dataset used and/or analyzed in the current study is available from the corresponding author on reasonable request.

\section{Ethics approval and consent to participate}

The study has been approved by the Institutional Review Board of Beijing Chaoyang Hospital affiliated to Capital Medical University, Beijing. Written informed consents for receiving KA-TKA were obtained from all patients. This written informed consent includes surgical benefits, possible risks, and data related to KA-TKA may be used for any related retrospective clinical studies.

\section{Consent for publication}

Written informed consent for publication of their clinical details and/or clinical images was obtained from the patient.

\section{Competing interests}

The authors declare that they have no competing interests.

\section{Author details}

'Department of Orthopedic Surgery, Beijing Chaoyang Hospital, Capital Medical University, 100020 Beijing, China. ${ }^{2}$ Beijing Naton Medical Technology Innovation Center Co., Ltd, 100038 Beijing, China. ${ }^{3}$ The Center of Diagnosis and Treatment for Joint Disease, Rehabilitation Research Center, 100068 Beijing, China.
Received: 28 September 2020 Accepted: 9 February 2021

Published online: 17 February 2021

\section{References}

1. Passias PG, Bono OJ, Bono JV. Total knee arthroplasty in patients of advanced age: a look at outcomes and complications. J Knee Surg. 2020; 33(1):1-7.

2. Lange JK, Lee YY, Spiro SK, Haas SB. Satisfaction rates and quality of life changes following total knee arthroplasty in age-differentiated cohorts. J Arthroplast. 2018;33(5):1373-8.

3. Bellemans J, Colyn W, Vandenneucker H, Victor J. The Chitranjan Ranawat award: is neutral mechanical alignment normal for all patients? The concept of constitutional varus. Clin Orthop Relat Res. 2012;470(1):45-53.

4. Maderbacher G, Keshmiri A, Krieg B, Greimel F, Grifka J, Baier C. Kinematic component alignment in total knee arthroplasty leads to better restoration of natural tibiofemoral kinematics compared to mechanic alignment. Knee Surg Sports Traumatol Arthroscopy. 2019;27(5):1427-33.

5. Vendittoli PA, Blakeney W. Redefining knee replacement. Orthopaedics Traumatol Surg Res. 2017;103(7):977-9.

6. Theodore W, Twiggs J, Kolos E, Roe J, Fritsch B, Dickison D, Liu D, Salmon L, Miles B, Howell S. Variability in static alignment and kinematics for kinematically aligned TKA. Knee. 2017;24(4):733-44.

7. Konan S, Howell S, Oussedik S. Alignment targets in total knee arthroplasty. In: Total knee arthroplasty. Springer; 2015. p. 145-159.

8. Shelton TJ, Gill M, Athwal G, Howell SM, Hull ML. Outcomes in patients with a calipered kinematically aligned TKA that already had a contralateral mechanically aligned TKA. J Knee Surg. 2021;34(1):87-93.

9. Kim KK, Howell SM, Won YY. Kinematically aligned total knee arthroplasty with patient-specific instrument. Yonsei Med J. 2020;61(3):201-9.

10. Niki Y, Nagura T, Kobayashi S, Udagawa K, Harato K. Who will benefit from kinematically aligned total knee arthroplasty? Perspectives on patientreported outcome measures. J Arthroplasty. 2020;35(2):438-42.

11. Riviere C, Iranpour F, Auvinet E, Howell S, Vendittoli PA, Cobb J, Parratte S. Alignment options for total knee arthroplasty: A systematic review. Orthopaed Traumatol Surg Res. 2017;103(7):1047-56.

12. Nedopil AJ, Howell SM, Hull ML. What clinical characteristics and radiographic parameters are associated with patellofemoral instability after kinematically aligned total knee arthroplasty? Int Orthopaedics. 2017;41(2): 283-91.

13. Koh IJ, Park IJ, Lin CC, Patel NA, Chalmers CE, Maniglio M, McGarry MH, Lee TQ. Kinematically aligned total knee arthroplasty reproduces native patellofemoral biomechanics during deep knee flexion. Knee Surg Sports Traumatol Arthrosc. 2019;27(5):1520-8.

14. Keshmiri A, Maderbacher G, Baier C, Benditz A, Grifka J, Greimel F. Kinematic alignment in total knee arthroplasty leads to a better restoration of patellar kinematics compared to mechanic alignment. Knee Surg Sports Traumatol Arthrosc. 2019;27(5):1529-34.

15. Riviere C, Dhaif F, Shah H, Ali A, Auvinet E, Aframian A, Cobb J, Howell S, Harris S. Kinematic alignment of current TKA implants does not restore the native trochlear anatomy. Orthopaed Traumatol Surg Res. 2018; 104(7):983-95.

16. Ishikawa M, Kuriyama S, Ito H, Furu M, Nakamura S, Matsuda S. Kinematic alignment produces near-normal knee motion but increases contact stress after total knee arthroplasty: A case study on a single implant design. Knee. 2015;22(3):206-12.

17. Maillot C, Leong A, Harman C, Morelli A, Mospan R, Cobb J, Riviere C. Poor relationship between frontal tibiofemoral and trochlear anatomic parameters: Implications for designing a trochlea for kinematic alignment. Knee. 2019;26(1):106-14.

18. Rivière C, Iranpour F, Auvinet E, Aframian A, Asare K, Harris S, Cobb J, Parratte S. Mechanical alignment technique for TKA: Are there intrinsic technical limitations? Orthopaed Traumatol: Surg Res. 2017;103(7):1057-67.

19. Kawahara S, Okazaki K, Okamoto S, Iwamoto Y, Banks SA. A lateralized anterior flange improves femoral component bone coverage in current total knee prostheses. Knee. 2016;23(4):719-24.

20. Kim JT, Han J, Shen QH, Moon SW, Won YY. Morphological patterns of anterior femoral condylar resection in kinematically and mechanically aligned total knee arthroplasty. J Arthroplast. 2018;33(8):2506-11.

21. Kellgren JH, Lawrence JS. Radiological assessment of osteo-arthrosis. Ann Rheum Dis. 1957;16(4):494-502. 
22. Howell SM, Papadopoulos S, Kuznik KT, Hull ML. Accurate alignment and high function after kinematically aligned TKA performed with generic instruments. Knee Surg Sports Traumatol Arthrosc. 2013;21 (10):2271-80.

23. Paley D, Herzenberg JE, Tetsworth K, McKie J, Bhave A. Deformity planning for frontal and sagittal plane corrective osteotomies. Orthopedic Clin North America. 1994;25(3):425-65.

24. Walter SD, Eliasziw M, Donner A. Sample size and optimal designs for reliability studies. Stat Med. 1998;17(1):101-10

25. Yoshino K, Hagiwara S, Nakamura J, Tsukeoka T, Tsuneizumi Y, Ohtori S. Intra- and interobserver reliability and agreement in three-dimensional computed tomography measurements of component positions after total knee arthroplasty. Knee. 2019;26(5):1102-10.

26. Brar AS, Howell SM, Hull ML, Mahfouz MR. Does kinematic alignment and flexion of a femoral component designed for mechanical alignment reduce the proximal and lateral reach of the trochlea? J Arthroplast. 2016;31(8): 1808-13.

27. Howell SM, Howell SJ, Hull ML. Assessment of the radii of the medial and lateral femoral condyles in varus and valgus knees with osteoarthritis. J Bone Joint Surg Am. 2010;92(1):98-104.

28. Eckhoff D, Hogan C, DiMatteo L, Robinson M, Bach J. Difference between the epicondylar and cylindrical axis of the knee. Clin Orthop Relat Res. 2007; 461:238-44.

29. Merican AM, Ghosh KM, Iranpour F, Deehan DJ, Amis AA. The effect of femoral component rotation on the kinematics of the tibiofemoral and patellofemoral joints after total knee arthroplasty. Knee Surg Sports Traumatol Arthrosc. 2011:19(9):1479-87.

30. Marra MA, Strzelczak M, Heesterbeek PJC, van de Groes SAW, Janssen D, Koopman B, Verdonschot N, Wymenga AB. Flexing and downsizing the femoral component is not detrimental to patellofemoral biomechanics in posterior-referencing cruciate-retaining total knee arthroplasty. Knee Surg Sports Traumatol Arthrosc. 2018;26(11):3377-85.

31. Meier M, Zingde S, Steinert A, Kurtz W, Koeck F, Beckmann J. What is the possible impact of high variability of distal femoral geometry on TKA? A CT data analysis of 24,042 knees. Clin Orthop Relat Res. 2019;477(3):561-70.

\section{Publisher's Note}

Springer Nature remains neutral with regard to jurisdictional claims in published maps and institutional affiliations.

Ready to submit your research? Choose BMC and benefit from:

- fast, convenient online submission

- thorough peer review by experienced researchers in your field

- rapid publication on acceptance

- support for research data, including large and complex data types

- gold Open Access which fosters wider collaboration and increased citations

- maximum visibility for your research: over $100 \mathrm{M}$ website views per year

At $\mathrm{BMC}$, research is always in progress.

Learn more biomedcentral.com/submissions 